\title{
gु
}

\section{Nonlinear Inverse Synthesis and Eigenvalue Division Multiplexing in Optical Fiber Channels}

\author{
Jaroslaw E. Prilepsky, ${ }^{1 *}$ Stanislav A. Derevyanko, ${ }^{2}$ Keith J. Blow, ${ }^{1}$ Ildar Gabitov, ${ }^{3}$ and Sergei K. Turitsyn ${ }^{1}$ \\ ${ }^{1}$ Aston Institute of Photonic Technologies, Aston University, B4 7ET Birmingham, United Kingdom \\ ${ }^{2}$ Department of Physics of Complex Systems, Weizmann Institute of Science, Rehovot 76100, Israel \\ ${ }^{3}$ Department of Mathematics, University of Arizona, 617 North Santa Rita Avenue, \\ P.O. Box 210089, Tucson, Arizona 85721, USA
}

(Received 13 February 2014; published 1 July 2014)

\begin{abstract}
We scrutinize the concept of integrable nonlinear communication channels, resurrecting and extending the idea of eigenvalue communications in a novel context of nonsoliton coherent optical communications. Using the integrable nonlinear Schrödinger equation as a channel model, we introduce a new approachthe nonlinear inverse synthesis method-for digital signal processing based on encoding the information directly onto the nonlinear signal spectrum. The latter evolves trivially and linearly along the transmission line, thus, providing an effective eigenvalue division multiplexing with no nonlinear channel cross talk. The general approach is illustrated with a coherent optical orthogonal frequency division multiplexing transmission format. We show how the strategy based upon the inverse scattering transform method can be geared for the creation of new efficient coding and modulation standards for the nonlinear channel.
\end{abstract}

Optical fiber systems form the backbone of the global telecommunication networks and currently carry the majority of the world's information traffic, with the "fifth generation" of optical transmission systems operating with advanced modulation formats, e.g., orthogonal frequency division multiplexing (OFDM), digital signal processing techniques, etc. [1]. Rapidly increasing data rates in the core fiber communication systems are currently approaching the limits of transmission technologies, many of which were originally developed for linear (radio) communication channels [2]. The paramount limit on channel spectral efficiency is imposed by fiber nonlinearity [2-8]. Therefore, the applications of "linear techniques" in a realistic fiber channel are limited by the nonlinear properties of the fiber medium itself. The increase in the signal power to improve the signal-to-noise ratio (SNR) leads to power-dependent nonlinear transmission distortions. Thus, nonlinearity is an essential component in the design of advanced fiber communication systems, but it is often shunned by engineers in view of its complexity. There is an evident need for radically different approaches to coding, transmission, and processing of information in fiber communication channels that would take into account the nonlinear properties of the optical fiber. In this Letter, we present a nonlinear inverse synthesis (NIS) method-a highly promising alternative transmission technique based on the integrability of the nonlinear channel.

Published by the American Physical Society under the terms of the Creative Commons Attribution 3.0 License. Further distribution of this work must maintain attribution to the author(s) and the published article's title, journal citation, and DOI.
The evolution of light in an optical fiber is modeled by the nonlinear Schrödinger equation (NLSE) [2,4], which accounts for the interplay between dispersion and nonlinearity. Averaging over periodic gain and loss variation in practical systems leads to effectively conservative signal dynamics governed by the renormalized NLSE [9]. Moreover, a recent experimental demonstration of a quasilossless fiber span [10] shows that gain (loss) variations can be compensated continuously along the fiber. Importantly, the NLSE (without perturbations) belongs to the class of the integrable nonlinear systems [9,11-14]. The integrability of the NLSE means that one can present nonlinear field evolution in a special basis, within which the dynamics of individual "nonlinear normal modes" is effectively linear. A powerful method - the inverse scattering transform (IST) $[9,12-14]$, that is a nonlinear analog of the Fourier transform [14] — can be applied to find the solution of an integrable equation. Within this method, the first step (decomposition of initial conditions into spectral data) consists of solving a linear spectral Zakharov-Shabat problem (ZSP) [12]. Any field evolving according to the NLSE can be described by a set of scattering data evolving in a linearlike manner, this fact is the foundation of the nonlinear Fourier transform (NFT) method [9,12-14]. The NFT does to the NLSE what the FT does to the linear equations: Just as the linear FT changes dispersion to a phase rotation in frequency space so the NFT leads to a trivial phase rotation of the spectral data. Thus, the fiber nonlinear effects are effectively included in the NFT $[9,12-14]$. This property constitutes the general idea of the eigenvalue communication introduced first in [15], the essence of which is to use invariant ZSP eigenvalues to encode and transmit information [16]. The use of NFT 
decomposition opens fundamentally new possibilities for advanced coding and modulation schemes, resistant to nonlinear fiber effects.

Note that, in Ref. [15], only the discrete part of the ZSP spectrum was considered. This discrete spectrum corresponds to the soliton part of the NLSE solution [9,12-14]. On the other hand, the vast amount of available degrees of freedom contained in the continuous part of the spectral data has been generally overlooked so far. In recent years, the concept of transmission built on pure fundamental NLSE solitons has lost much of its appeal in contemporary wavelength division multiplexing systems due to problems caused by soliton collisions and corresponding cross talk $[9,17]$ (though a recent study [18] indicated mechanisms for the mitigation of intersoliton cross talk). Very recently there has arisen a considerable renewed interest in transmission based on the integrability and IST principles [19-21], where, not the solitons themselves, but the IST spectral data associated with each solitary degree of freedom were utilized. In this Letter, we, however, apply the idea of integrability in a completely novel context of nonsoliton coherent optical communications. We introduce a new approach to digital signal processing based on the encoding of information directly onto the continuous nonlinear signal spectrum that evolves linearly along the transmission line in a nonlinear integrable channel. We call this approach nonlinear eigenvalue division multiplexing. The main result reported here is that, by applying the IST technique, one can develop a straightforward signal processing routine for compensating nonlinear distortions of arbitrary magnitude.

We consider, as the master model, the NLSE with constant anomalous dispersion, describing the propagation of a slowly varying optical field envelope $q(z, t)$ inside a single-mode nonlinear fiber [2]

$$
i q_{z}+\frac{1}{2} q_{t t}+q|q|^{2}=\eta(z, t),
$$

where $z$ stands for the propagation distance and $t$ is the time in the frame comoving with the envelope velocity. In Eq. (1), time is normalized to the characteristic duration of our input signal $T_{s}$, distance-by the dispersion length: $Z_{s}=T_{s}^{2} /\left|\beta_{2}\right|$, $\beta_{2}=-21.67 \mathrm{ps}^{2} / \mathrm{km}$ is the dispersion coefficient; the power is measured in units of $\left(\gamma Z_{s}\right)^{-1}$, with the nonlinearity coefficient $\gamma=1.27(\mathrm{~W} \mathrm{~km})^{-1}$. The random quantity $\eta(t, z)$ describes the noise generation due to amplifier spontaneous emission (ASE) [2]: It is a symmetric additive complex Gaussian white noise with zero average, fully characterized by its autocorrelation intensity: $\left\langle\eta(t, z) \bar{\eta}\left(t^{\prime}, z^{\prime}\right)\right\rangle=$ $2 D \delta\left(t-t^{\prime}\right) \delta\left(z-z^{\prime}\right)$ [the overbar means complex conjugation]. This model describes the amplification scheme, in which the distributed Raman gain exactly compensates for the fiber loss: Refs. [2] provide a detailed account of how noise intensity $D$ is related to the line parameters.

As the NLSE with zero right-hand side is integrable, the explicit procedures for the forward and backward NFT are known [9,12-14]. The decomposition of the signal into spectral data (forward NFT) is achieved by solving the ZSP equations for auxiliary functions $v_{1}, v_{2}$

$$
\frac{d v_{1}}{d t}=q(t) v_{2}-i \zeta v_{1}, \quad \frac{d v_{2}}{d t}=-\bar{q}(t) v_{1}+i \zeta v_{2},
$$

where the input pulse shape $q(t)$ acts as a potential. Here $\zeta$ is a (generally complex) eigenvalue, $\zeta=\xi+i \varrho$, and $q(t)$ decays as $t \rightarrow \pm \infty$. To define scattering data, for real $\zeta=\xi$, one fixes two linearly independent Jost solutions of Eq. (2), $\Phi(t, \xi)=\left[\phi_{1}, \phi_{2}\right]^{T}$, and $\tilde{\Phi}(t, \xi)=\left[\bar{\phi}_{2},-\bar{\phi}_{1}\right]^{T}$, with the condition at the left end: $\left.\Phi\right|_{t \rightarrow-\infty}=\left[e^{-i \xi t}, 0\right]^{T}$; in the same manner, we fix two other Jost solutions, $\Psi(t, \xi)=\left[\psi_{1}, \psi_{2}\right]^{T}$ and $\tilde{\Psi}(t, \xi)=\left[\bar{\psi}_{2},-\bar{\psi}_{1}\right]^{T}$, at the right end: $\Psi_{t \rightarrow \infty}=\left[0, e^{i \xi t}\right]$. These two sets are linearly dependent and relate through the Jost scattering coefficients $a(\xi)$ and $b(\xi): \Phi=a \tilde{\Psi}+b \Psi$, $\tilde{\Phi}=-\bar{a} \Psi+\bar{b} \tilde{\Psi}$. The (left) reflection coefficient is defined as $r(\xi)=\bar{b}(\xi) / a(\xi)$, and solitons correspond to the complex eigenvalues $\zeta_{n}$, where $a\left(\zeta_{n}\right)=0$. The forward NFT operation corresponds to mapping of the initial field, $q(0, t)$, onto a set of scattering data: $\Sigma=\left[\{r(\xi), \xi \in \mathbb{R}\} ;\left\{\zeta_{n}, \gamma_{n}=\right.\right.$ $\left.\left.\left[b\left(\zeta_{n}\right) a_{\zeta}{ }^{\prime}\left(\zeta_{n}\right)\right]^{-1}\right\}\right]$, where the index $n$ runs over all discrete eigenvalues of ZSP; $r(\xi)$ plays the role of the nonlinear spectral profile. The nonlinear spectral function (NS) defined as $N(\omega)=-\left.r(\xi)\right|_{\xi=-\omega / 2}$, serves as the direct nonlinear analogue of the Fourier spectrum, tending to the ordinary FT of $q(t)$ in the linear limit [22]. The evolution of $r(\xi)$ is trivial: $r(\xi, L)=r(\xi) e^{-2 i \xi^{2} L}$ (with $L$ being the propagation distance), and substituting this expression into the definition of NS, one finds that $N(\omega)$ obeys the linear dispersion law of the NLSE. Therefore, the orthogonality of nonlinear normal modes is preserved during signal propagation within the NLSE.

The backward NFT maps the scattering data $\Sigma$ onto the field $q(t)$ : This is achieved via the Gelfand-LevitanMarchenko equation (GLME) for the unknown function $K\left(t, t^{\prime}\right)$ [9,12-14]. For the soliton-free case, considered further in our study, the GLME can be written as

$$
\begin{aligned}
& K\left(t, t^{\prime}\right)+F\left(t+t^{\prime}\right) \\
& \quad+\iint_{-\infty}^{t} K(t, \lambda) \bar{F}(\lambda+\sigma) F\left(\sigma+t^{\prime}\right) d \sigma d \lambda=0 .
\end{aligned}
$$

Here $F(t)=(2 \pi)^{-1} \int d \xi r(\xi) e^{-i \xi t}$ is the linear backward FT of $r(\xi)$; having solved the GLME (3) for $K\left(t, t^{\prime}\right)$, the sought solution in the space-time domain is recovered as $q(t)=2 K(t, t)[22]$.

Transmission using the NIS.-The preliminary "proof of concept" demonstration that the nonsolitonic part of NS can be used for mitigating nonlinear distortions was presented in Refs. [21,23]. However, these methods have limited applicability being confined to low-power initial signals or to the normal dispersion case, where no solitons generally appear. The case studied in our work is rather more challenging and practically important: For the focusing NLSE, the eigenvalue spectrum of ZSP generally brings 
about complex discrete eigenvalues corresponding to solitons; when the input is randomly coded, the eigenvalue portrait of the ZSP can be extremely involved.

To get rid of this issue, we suggest synthesizing the profile in the time domain starting from given encoded shapes in the nonlinear spectral domain (see also discussion in the Supplemental Material [24]). This idea is similar to that widely used for the syntheses of corrugated waveguides [25] and Bragg gratings [26,27]: one creates the input profile having the desired properties, starting from the nonlinear spectral data, and then, employs the backward NFT synthesizing the profile in the time domain. During the evolution, the spectral data undergo just a phase rotation, and hence, after unrolling this "nonlinear dispersion" at the receiver, the initial information can be recovered without nonlinear signal degradation. The scheme of the NIS method vs the simple conventional transmission is illustrated in Fig. 1; we show the true profiles for the transmission of a truncated harmonic: $q(t)=e^{i \Omega t}, t \in[0, T], \Omega=2 \pi / T$. The NIS method involves two stages, see Fig. 1(b): (i) The backward NFT at the transmitter, providing the profile $q_{\mathrm{GLM}}(0, t)$ in the time domain corresponding to a desired initial NS $N(\omega)$; (ii) Recovery of the NS at the receiver by the forward NFT, i.e., by solving Eq. (2), and consequent linear dispersion removal (the latter is for recovering the initial phase information). For the conventional transmission, Fig. 1(a), we also removed the linear dispersion at the receiver. One observes, that while the profile for the conventional transmission is heavily degraded by the nonlinearity, the recovery of the initial waveform after using the NIS method is perfect.

We now discuss the numerical complexity of the NIS as compared to the popular digital back propagation (DBP) method for the removal of the nonlinear distortions [28]. In
DBP, one reads the waveform at the receiver, inserts it in the noiseless NLSE, and then solves the NLSE in the backward direction. The numerical method for the NLSE integration is usually the split-step Fourier method [4,28], which requires $N_{z} M_{t} \log M_{t}$ operations; $M_{t}$ being the number of discretization points in time, and $N_{z}$ - the number of steps in $z$, that grows with the transmission length and pulse power. In realistic transmission problems, one typically has $N_{z} \gg 1$. The NIS method involves just two nonlinear transforms, each of those requiring $\sim M_{t}^{2}$ operations for well-developed methods [27,29]. Even with such an estimate, the complexity of the NIS can already be comparable to that of the DBP [21]. However, the recent advancements in numerical NFT indicate that the complexity of NIS can be reduced even further. Namely, one of the fastest methods for the GLME solution is based on a single inversion of a Toeplitz matrix [27]. Using the newest algorithms [30], this inversion can be performed in just $M_{t} \log M_{t}$ operations. For the ZSP, a recent study [31] suggests, that the recovery of continuous NS can be made in only $\sim M_{t} \log ^{2} M_{t}$ operations. With these estimates in mind, we believe that NIS-based transmission methods can significantly outperform the DBP in terms of numerical complexity for the digital signal processing.

To illustrate how NIS performs in a realistic digital transmission scheme, we consider the popular OFDM encoding [32] of the input waveform both in the linear and nonlinear spectral domain. The first question concerning the NIS implementation is what do the elementary OFDM base functions look like when its spectral shape is used in the NS domain? We present our results for two kinds of base functions in Fig. 2(a)-2(d). Figs. 2(a) and 2(b) show the results for the sinc base (a rectangle in the $\omega$ domain). This

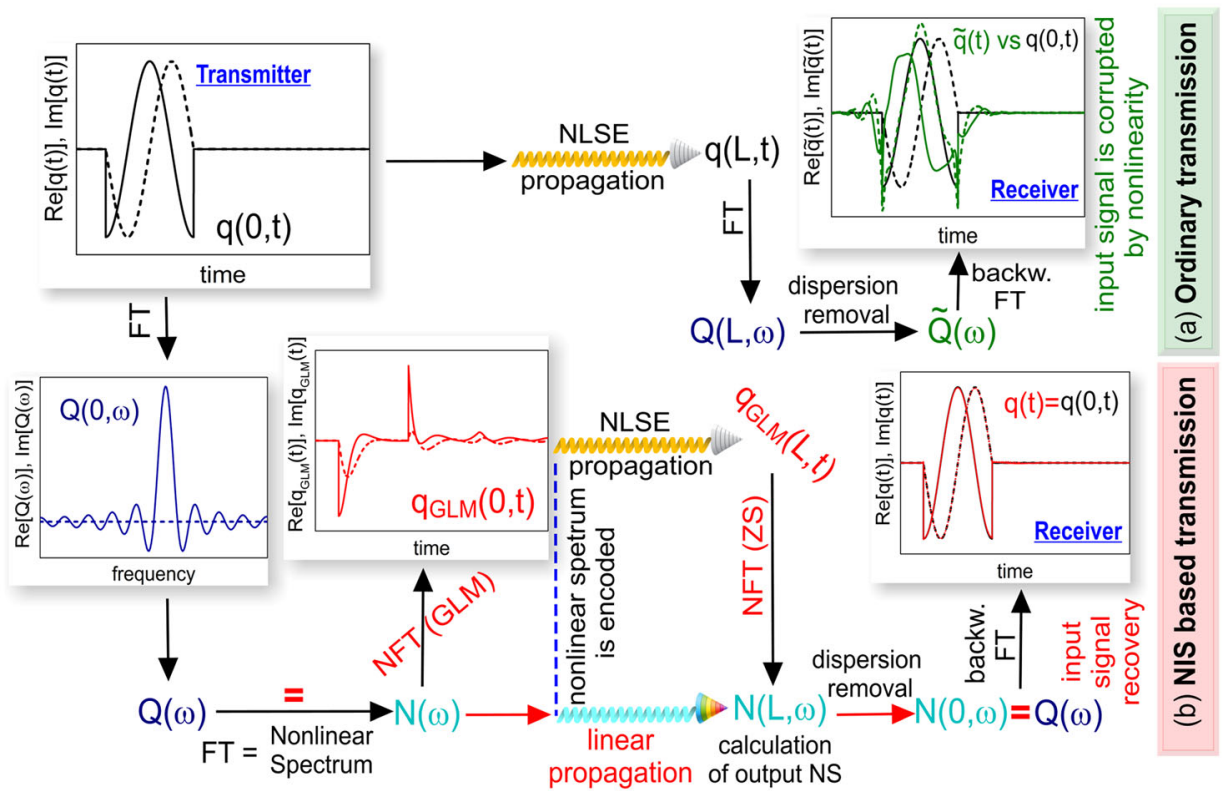

FIG. 1 (color online). The flow chart depicting the operations for an example initial waveform $q(0, t)=e^{2 \pi i t / T}$ if $t \in[0, T], 0$ otherwise. The upper part (a) shows the conventional transmission scheme, and the lower part (b) corresponds to the NIS-based transmission. (a),(b) display the true profiles for $T=1 \mathrm{~ns}$, transmission length $L=2000 \mathrm{~km}$ (noiseless case). 

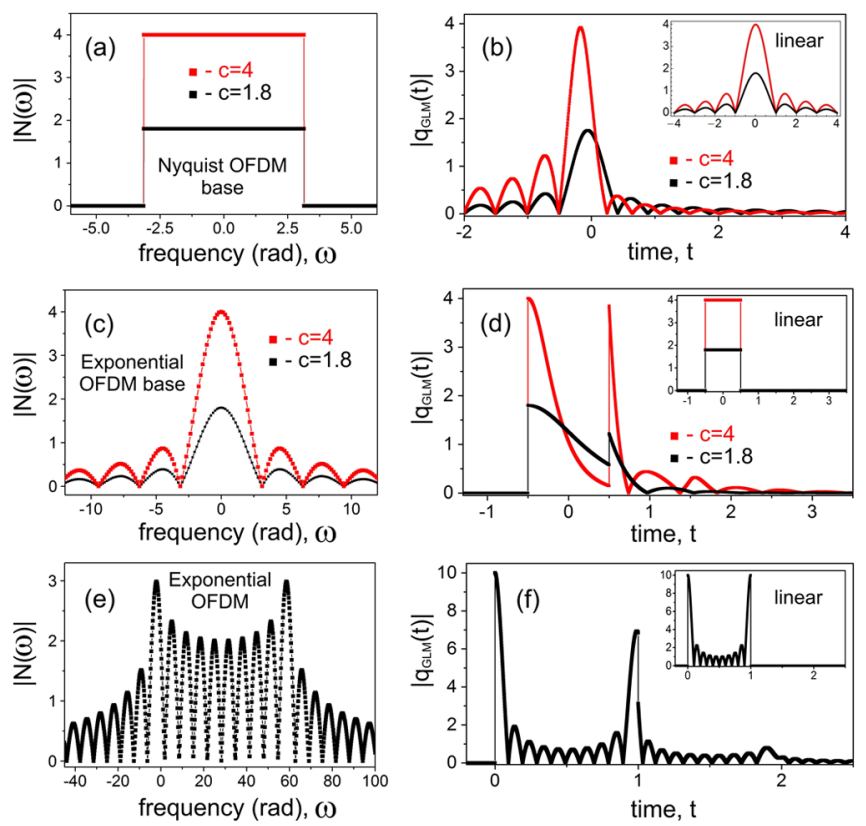

FIG. 2 (color online). The profiles of NS $|N(\omega)|$ with different amplitudes, and the corresponding profiles $\left|q_{\mathrm{GLM}}(t)\right|$ in the time domain, obtained by solving GLM equation (3). (a),(b) for the rect-shaped $N(\omega)=\operatorname{rect}(\omega / 2 \pi)$ (Nyquist OFDM); (c),(d) for the sinc-shaped $N(\omega)$ (standard exponential-based OFDM); (e), (f) $N(\omega)$ and corresponding $q_{\mathrm{GLM}}(t)$ for a single slot of exponential-based OFDM (10 modes were used), encoded onto the NS, Eq. (4), with $c_{j k}=1$. The insets show the corresponding linear FTs of the spectrum.

base function is utilized in the Nyquist-format technique, which provides the highest spectral efficiency [33]. As seen in Fig. 2(b), the corresponding profiles in the time domain, $q_{\mathrm{GLM}}(t)$, are already not symmetric, and the asymmetry grows with the increase of $c$ (see the Supplemental Material [24] for analytical considerations). In the OFDM scheme, the elementary base in the time domain is simply a rectangle (i.e., a sinc function in the $\omega$ domain) and, without loss of generality, in what follows, we will focus on this format [24]. The inverse NFT of the single OFDM spectral tone, $N(\omega)=c \operatorname{sinc}(\omega)$ is given in Fig. 2(c), (d) for different values of amplitude $c$. We see that for a sufficiently large $c$ the waveform of $q_{\mathrm{GLM}}(t)$ is significantly different from a rectangular profile occurring in the linear case: While losing its symmetry, the profile develops an oscillatory advancing tail. The general form of the spectrum of an arbitrary OFDMencoded data sequence is [32]

$$
Q(\omega)=\sum_{j=-\infty}^{\infty} \sum_{k=1}^{N_{\mathrm{sc}}} c_{j k} \operatorname{sinc}\left(\frac{\Omega_{k}-\omega}{2}\right) e^{i\left(\Omega_{k}-\omega\right) j},
$$

where $c_{j k}$ is the $j$ th informational symbol in the $k$ th subcarrier, $N_{\mathrm{sc}}$ is the total number of subcarriers, $\Omega_{k}$ is the frequency of the $k$ th subcarrier. The frequencies are spaced at multiples of the inverse of the symbol rate: $\Omega_{k}=2 \pi(k-1)$ (normalized slot duration $T_{s}=1$ ), which ensures the orthogonality of subcarriers. In Figs. 2(e)-2(f) we present the spectrum of a single OFDM slot with 10 subcarriers with $c_{k}=1$, and a corresponding inverse NFT, $q_{\mathrm{GLM}}(t)$. In this case, the structure of the advancing tail is more involved and reflects the structure of the pulse itself.

For illustrative reasons, we now employ random quadrature phase shift keying (QPSK) encoding of the coefficients $c_{j k}$ [34]: The absolute value of $c_{j k}$ is the same for each coefficient, $\left|c_{j k}\right|=c$, and the phase of each $c_{j k}$ randomly takes one of four discrete values $\operatorname{Arg}\left\{c_{j k}\right\}=$ $\phi_{j k}=2 \pi p / 4, p=0, \ldots, 3$. Let us turn to the performance of the digital NIS transmission as opposed to the ordinary one, Fig. 1, using the inverse linear FT of Eq. (4), $q(t)$, as an input for the ordinary transmission, and its inverse NFT counterpart, $q_{\mathrm{GLM}}(t)$, for the NIS transmission. We quantify the noise influence on the transmission by optical signal-tonoise ratio (OSNR), defined as OSNR $=P_{s} / 2 D L B_{\text {ref }}$ [2,32], where $P_{s}$ is the average power of the input signal and $B_{\text {ref }}$ is a reference bandwidth (it is the simulation bandwidth in our case). The lower the value of OSNR, the stronger is the signal degradation by the noise. We show the results of our simulations on the constellation diagram indicating positions of the normalized coefficients $C_{j k}=$ $c_{j k} / c$ after the transmission. For ordinary transmission, we use the scheme given in Fig. 1(a), the coefficients are recovered by a linear FT in the time domain. For NIS transmission, the scheme in Fig. 1(b) is used: The QPSKOFDM coefficients are recovered from the NS domain. Our results are given in Fig. 3 for the OSNR $=7 \mathrm{~dB}$ (medium noise). We observe that the conventional transmission performs poorly leading to an almost complete smearing of the constellation picture. In contrast, the locus of coefficients $C_{j k}$ for the NIS-based transmission is still close to the initial values (blue rectangles), indicating that the NIS method significantly outperforms its linear counterpart. The plots for different OSNR levels and lower input power are given in the Supplemental Material [24], indicating that our results are general. In Figs. 3(b)-3(c) we present the probability density functions (PDFs) for the angular and radial distributions of $C_{j k}$ : These distributions also demonstrate the superior performance of the NIS as compared to the ordinary transmission.

In this Letter, we have demonstrated that the nonlinear spectral data can be used for high-quality long-haul information transmission. The advantage of using the NS is that it plays the role of the linear spectrum for the nonlinear integrable problem, and the encoded spectral content undergoes just the trivial phase rotation obeying the linear dispersion law. We have introduced the nonlinear inverse synthesis method that has its roots in the theory of fiber Bragg grating synthesis and consists in the direct encoding of the nonlinear spectral domain followed by reconstruction of the field profile in the time domain by solving the GLME. We illustrated the robustness of the NSbased transmission not only against nonlinearity, but also against the ASE noise using a popular OFDM modulation format as an example. 

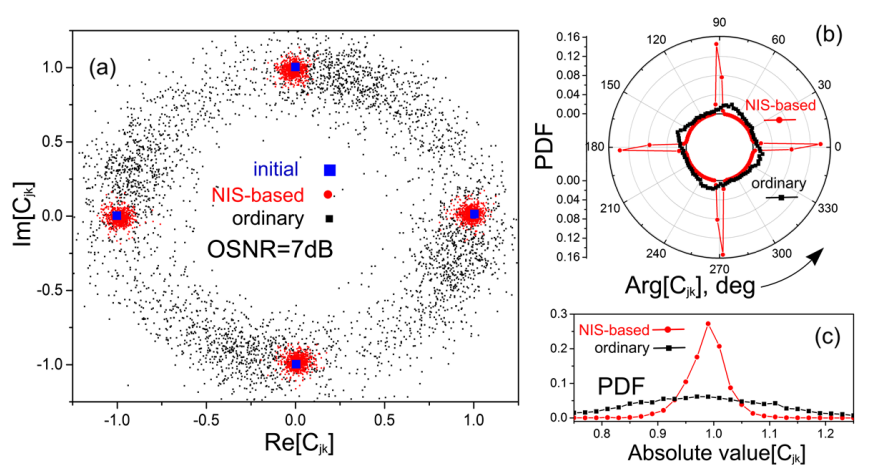

FIG. 3 (color online). Comparison of the digital QPSK-OFDM propagation (with 10 tones in each slot, $\left|c_{j k}\right|=c=0.9$ ) based on the NIS vs the ordinary one, see Fig. 1; the propagation distance $L \approx 2200 \mathrm{~km}$, OSNR $=7 \mathrm{~dB}$. (a) Constellation diagram for normalized OFDM coefficients $C_{j k}$ with QPSK coding. (b) PDFs for the $\operatorname{Arg}\left[C_{j k}(L)\right]$ for the NIS and ordinary transmission. (c) PDFs for $\left|C_{j k}(L)\right|$ for the NIS and ordinary transmission.

The work has been supported by the EPSRC Project UNLOC No. EP/J017582/1. The support of the Russian Ministry of Education and Science, European Research Council, and Marie Curie IRSES program is acknowledged. The authors are thankful to Sander Wahls for kindly sending us the results concerning the superfast forward NFT prior to their publication. We are also indebted to David Shapiro and Leonid Frumin for helpful comments with regard to the numerical solution of GLME.

*y.prylepskiy1@aston.ac.uk

[1] M. Cvijetic and I. B. Djordjevic, Advanced Optical Communication Systems and Networks, (Artech House, Norwood, MA, 2013).

[2] R.-J. Essiambre, G. Kramer, P. J. Winzer, G. J. Foschini, and B. Goebel, J. Lightwave Technol. 28, 662 (2010); R.-J. Essiambre, G. J. Foschini, G. Kramer, and P. J. Winzer, Phys. Rev. Lett. 101, 163901 (2008); R.-J. Essiambre, G. J. Foschini, G. Kramer, and P. J. Winzer, Bell Labs Tech. J. 14, 149 (2010).

[3] P. P. Mitra and J. B. Stark, Nature (London) 411, 1027 (2001).

[4] G.P. Agrawal, Fiber-Optic Communication Systems (Wiley, Hoboken, NJ, 2010), 4th ed..

[5] A. D. Ellis, J. Zhao, and D. Cotter, J. Lightwave Technol. 28, 423 (2010).

[6] D. J. Richardson, Science 330, 327 (2010).

[7] R. I. Killey and C. Behrens, J. Mod. Opt. 58, 1 (2011).

[8] K. S. Turitsyn, S. A. Derevyanko, I. V. Yurkevich, and S. K. Turitsyn, Phys. Rev. Lett. 91, 203901 (2003).

[9] A. Hasegawa and Y. Kodama, Solitons in Optical Coтmunications (Oxford University Press, Oxford, 1995).

[10] J. D. Ania-Castanon, T. Ellingham, R. Ibbotson, X. Chen, L. Zhang, and S. Turitsyn, Phys. Rev. Lett. 96, 023902 (2006).

[11] Yu. S. Kivshar and G. P. Agrawal, Optical Solitons: From Fibers to Photonic Crystals (Academic Press, San Diego, 2003).

[12] V. E. Zakharov and A. B. Shabat, Sov. Phys. JETP 34, 62 (1972).
[13] A. C. Newell, Solitons in Mathematics and Physics. (SIAM, Philadelphia, 1985); V.E. Zakharov, S. V. Manakov, S. P. Novikov, and L. P. Pitaevskii, Theory of Solitons. The Inverse Scattering Method (Consultants Bureau, New York, 1984).

[14] M. J. Ablowitz, D. J. Kaup, A. C. Newell, and H. Segur, Stud. Appl. Math. 53, 249 (1974).

[15] A. Hasegawa and T. Nyu, J. Lightwave Technol. 11, 395 (1993).

[16] S. Oda, A. Maruta, and K. Kitayama, IEEE Photonics Technol. Lett. 16, 587 (2004); H. Terauchi and A. Maruta, in Proceedings of the 18th OptoElectronics and Communications Conference (IEEE, New York, 2013), paper WR2-5.

[17] A. Peleg, M. Chertkov, and I. Gabitov, J. Opt. Soc. Am. B 21, 18 (2004).

[18] J. E. Prilepsky, S. A. Derevyanko, and S. K. Turitsyn, Phys. Rev. Lett. 108, 183902 (2012); J. Phys. A 45, 025202 (2012).

[19] M. I. Yousefi and F. R. Kschischang, arXiv:1202.3653; , arXiv:1204.0830; arXiv:1302.2875.

[20] E. Meron, M. Feder, and M. Shtaif, arXiv:1207.0297.

[21] J. E. Prilepsky, S. A. Derevyanko, and S. K. Turitsyn, Opt. Express 21, 24344 (2013).

[22] Sometimes, instead of the left reflection coefficient, one uses its "right" counterpart $\tilde{r}(\xi)=b(\xi) / a(\xi)$. Clearly, both definitions have the same spectral density $|N(\omega)|^{2}$. The main difference is that the GLM equation with the right reflection coefficient is inverted around $+\infty$ rather than $-\infty$ as in Eq. (3), [14].

[23] E. G. Turitsyna and S. K. Turitsyn, Opt. Lett. 38, 4186 (2013).

[24] See Supplemental Material at http://link.aps.org/ supplemental/10.1103/PhysRevLett.113.013901 for comparison of the NIS and straight IST methods, the asymptotic formulas for the inverse NFT for the nonlinear Nyquist OFDM base, comparison of the linear and NIS-based nonlinear OFDM spectra, and OFDM coefficient dynamics for different OSNR and OFDM power levels.

[25] G.-H. Song, and S.-Y. Shin, J. Opt. Soc. Am. A, 2, 1905 (1985).

[26] R. Kashyap, Fiber Bragg Gratings (Academic Press, New York, 1999).

[27] O. V. Belai, L. L. Frumin, E. V. Podivilov, and D. A. Shapiro, J. Opt. Soc. Am. B 24, 1451 (2007).

[28] E. Ip and J. Kahn, J. Lightwave Technol. 26, 3416 (2008).

[29] S. Burtsev, R. Camassa, and I. Timofeyev, J. Comput. Phys. 147, 166 (1998); G. Boffetta and A. R. Osborne, ibid. 102, 252 (1992).

[30] M. Van Barel, G. Heinig, and P. Kravanja, SIAM J. Matrix Anal. Appl. 23, 494 (2001); S. Chandrasekaran, M. Gu, X. Sun, J. Xia, and J. Zhu, ibid. 29, 1247 (2008).

[31] S. Wahls and H. V. Poor, in Proceedings of International Conference on Acoustics, Speech, and Signal Processing (ICASSP 2013), (IEEE, New York, 2013), p. 5780; arXiv: 1402.1605.

[32] S. Shieh and I. Djordjevic, OFDM for Optical Communications, (Academic Press, New York, 2010).

[33] R. Schmogrow et al., Opt. Express 20, 317 (2012); M. A. Sotto, M. Alem, M. A. Shoaie, A. Vedadi, C.-S. Brès, L. Thévenaz, and T. Schneider, Nat. Commun. 4, 2898 (2013).

[34] A. H. Gnauk and P. J. Winzer, J. Lightwave Technol. 23, 115 (2005). 